Research Article

\title{
Generalization of $h$-Convex Stochastic Processes and Some Classical Inequalities
}

\author{
Hao Zhou, ${ }^{1}$ Muhammad Shoaib Saleem $\mathbb{D}^{2},{ }^{2}$ Mamoona Ghafoor, ${ }^{2}$ and Jingjing $\mathrm{Li}^{3}$ \\ ${ }^{1}$ Transportation School of Wuhan University of Technology, Wuhan University of Technology, Wuhan 430070, China \\ ${ }^{2}$ Department of Mathematics, University of Okara, Okara, Pakistan \\ ${ }^{3}$ China Ship Development and Design Center, Wuhan 430064, China
}

Correspondence should be addressed to Muhammad Shoaib Saleem; shaby455@yahoo.com

Received 30 April 2020; Revised 21 June 2020; Accepted 30 June 2020; Published 8 August 2020

Academic Editor: Shuanming Li

Copyright (@) 2020 Hao Zhou et al. This is an open access article distributed under the Creative Commons Attribution License, which permits unrestricted use, distribution, and reproduction in any medium, provided the original work is properly cited.

The field of stochastic processes is essentially a branch of probability theory, treating probabilistic models that evolve in time. It is best viewed as a branch of mathematics, starting with the axioms of probability and containing a rich and fascinating set of results following from those axioms. In probability theory, a convex function applied to the expected value of a random variable is always bounded above by the expected value of the convex function of the random variable. In this paper, the concept of generalized $h$-convex stochastic processes is introduced, and some basic properties concerning generalized $h$-convex stochastic processes are developed. Furthermore, we establish Jensen and Hermite-Hadamard and Fejér-type inequalities for this generalization.

\section{Introduction}

Stochastic processes are a branch of probability theory, treating probabilistic models that evolve in time [1-3]. It is a branch of mathematics, starting with the axioms of probability and containing a rich and fascinating set of results following from those axioms [4]. Although the results are applicable to many areas [5], they are best understood initially in terms of their mathematical structure and interrelationships [6].

There are various ways to define stochastic monotonicity and convexity for stochastic processes [7], and they are of great importance in optimization, especially in optimal designs, and also useful for numerical approximations when there exist probabilistic quantities in the literature [8]. We also refer [9-12] for detailed survey about the importance and interesting properties of stochastic models.

The idea of convex functions was put forward, and many generalizations were made in this area [13]. $h$-Convex [14] and $\phi$-convex functions [15] are famous generalizations of convex functions. Later, the theory of stochastic processes has developed very rapidly and has found application in a large number of fields. The study on convex stochastic processes was initiated in [16] by B. Nagy in 1974. After that, Nikodem in 1980 introduced the convex stochastic processes in his article [17]. Following this line of investigation, Skowronski described the properties of Jensen-convex and Wright-convex stochastic processes in $[18,19]$. Some interesting properties of convex and Jensen-convex processes are also presented in $[20,21]$. Assume a Jensen-convex stochastic process $\xi: I \times \Omega \longrightarrow \mathbb{R}$ which is mean-square continuous in $I$, where $I \subseteq \mathbb{R}$ is an interval. Then, for every $a_{1}, a_{2} \in I\left(a_{1}<a_{2}\right)$,

$$
\xi\left(\frac{a_{1}+a_{2}}{2}\right) \leq \frac{1}{a_{2}-a_{1}} \int_{a_{1}}^{a_{2}} \xi(u) \mathrm{d} u \leq \frac{\xi\left(a_{1}\right)+\xi\left(a_{2}\right)}{2}(\text { a.e. }),
$$

is known as the Hermite-Hadamard-type inequality for the convex stochastic process [22].

The aim of this paper is to introduce the notion of the generalized $h$-convex stochastic process and to extend the classical Hermite-Hadamard inequality for convex stochastic processes to generalized $h$-convex stochastic processes. 


\section{Novelty and Significance}

The study of convex functions makes them special because of their interesting properties as maximum are attained at the boundary point, and moreover, any local minimum is global one. So, this topic of research got the attention of many researchers of different areas because of their enormous applications in optimization theory. As far as convex stochastic processes are concerned, there is a lot of work in the last few decades. In [23], the authors investigated the gradient descent optimality for strongly convex stochastic optimization. A continuous-time financial portfolio selection model with expected utility maximization typically boils down to solve a convex stochastic optimization problem in terms of terminal wealth with budget constraints, see, e.g., [24]. For more details related to this work, see [25-31].

\section{Preliminaries}

Let $(\Omega, P)$ be a probability space. A function $\xi: \Omega \longrightarrow \mathbb{R}$ is a random variable if it is -measurable. A function $\xi: I \times \Omega \longrightarrow \mathbb{R}$, where $I \subseteq \mathbb{R}$ is an interval, is a stochastic process if for every $t \in I$, the function $\xi(t)$ is a random variable.

The stochastic process $\xi: I \times \Omega \longrightarrow \mathbb{R}$ is known as

(1) Stochastically continuous in $I$ if

$$
\mu-\lim _{v \longrightarrow v^{\circ}} \xi(v)=\xi\left(v^{\circ}\right)
$$

for all $v^{\circ} \in I$, where $\mu$ - lim represents the limit in the probability.

(2) Mean-square continuous in $I$ if

$$
\lim _{v \longrightarrow v^{\circ}} \mathbb{E}\left(\xi(v)-\xi\left(v^{\circ}\right)\right)^{2}=0,
$$

for all $v^{\circ} \in I$, where $\mathbb{E}[\xi(v)]$ represents an expectation value of random variable $\xi(v)$.

Clearly in probability, mean-square continuity implies continuity, but converse is not true.

A stochastic process $\xi$ is known as mean-square differentiable in $I$ if there is a stochastic process $\xi^{\prime}$ (derivative of $\xi)$ such that

$$
\lim _{v \longrightarrow v^{\circ}} \mathbb{E}\left[\frac{\xi(v)-\xi\left(v^{\circ}\right)}{v-v^{\circ}}-\xi^{\prime}\left(v^{\circ}\right)\right]^{2}=0,
$$

for every $v^{\circ} \in I$.

Now, we would like to recall the concept of the meansquare integral. For the definition and basic properties, see $[32,33]$.

Assume a stochastic process $\xi: I \times \Omega \longrightarrow \mathbb{R}$ with $\mathbb{E}\left[\xi(t)^{2}\right]<\infty$. A random variable $\zeta: \Omega \longrightarrow \mathbb{R}$ is said to be the mean-square integral of the process $\xi$ on $[a, b]$ if for every normal sequence of partitions of $\left[a_{1}, a_{2}\right]$, $a_{1}=v^{\circ}<v_{1}<\ldots<v_{n}=a_{2}$, and for all $\Theta_{k} \in\left[v_{k-1}, v_{k}\right]$, we have

$$
\lim _{n \longrightarrow \infty} \mathbb{E}\left[\left(\sum_{k=1}^{n} \xi\left(\Theta_{k}\right)\left(v_{k}-v_{k-1}\right)-\zeta(.)\right)^{2}\right]=0 .
$$

Then, we write

$$
\zeta(.)=\int_{a}^{b} \xi(s) \mathrm{d} s(\text { a.e }) .
$$

Now, we shall present some definitions and generalizations of the convex stochastic process.

Definition 1 (see [34]). A stochastic process $\xi: I \times \Omega \longrightarrow \mathbb{R}$ is known as generalized convex with respect to a bifunction $\eta: \xi(I) \times \xi(I) \longrightarrow \mathbb{R}$ if

$$
\xi(\alpha u+(1-\alpha) v) \leq \xi(v)+\alpha \eta(\xi(u), \xi(v))(\text { a.e }),
$$

for all $u, v \in I$ and $\alpha \in[0,1]$.

Definition 2 (see [35]). Let $h:(0,1) \longrightarrow \mathbb{R}$ be a function which is nonnegative and $h \neq 0$. A stochastic process $\xi: I \times$ $\Omega \longrightarrow \mathbb{R}$ is said to be $h$-convex if

$$
\xi(\alpha u+(1-\alpha) v) \leq h(\alpha) \xi(u)+h(1-\alpha) \xi(v)(\text { a.e }),
$$

for every $u, v \in I$ and $\alpha \in(0,1)$.

Definition 3 (see [15]). The function $\eta: \xi(I) \times \xi(I) \longrightarrow \mathbb{R}$ is said to be

(1) Nonnegatively homogeneous if $\eta(\gamma u, \gamma v)=\gamma \eta(u, v)$ for all $u, v \in \mathbb{R}$ and $\gamma \geq 0$

(2) Additive if $\eta\left(u_{1}, v_{1}\right)+\eta\left(u_{2}, v_{2}\right)=\eta\left(u_{1}+u_{2}, v_{1}+v_{2}\right)$ for all $u_{1}, u_{2}, v_{1}, v_{2} \in \mathbb{R}$

Definition 4 (see [14]). A function $h: J \longrightarrow \mathbb{R}, J \subseteq \mathbb{R}$, is said to be a supermultiplicative function if

$$
h(u v) \geq h(u) h(v)
$$

for all $u, v \in J$.

Now, we are ready to give our main definition.

Definition 5 (generalized $h$-convex stochastic process). $h$ and $\eta$ are fixed like above. We say that a stochastic process $\xi: I \times \Omega \longrightarrow$ is called generalized $h$-convex if

$$
\xi(\alpha u+(1-\alpha) v) \leq \xi(v)+h(\alpha) \eta(\xi(u), \xi(v))(\text { a.e }),
$$

for all $u, v \in I$ and $\alpha \in[0,1]$.

In (10), if we take $\eta(u, v)=u-v$ and $h(\alpha)=\alpha$, we obtain the convex stochastic process. We observe that, by taking $u=v$ in (10), we get

$$
h(\alpha) \eta(\xi(u), \quad \xi(u)) \geq 0,
$$

for any $u \in I$ and $\alpha \in[0,1]$, which implies that

$$
\eta(\xi(u), \quad \xi(u)) \geq 0,
$$

for any $u \in I$. Also, if we take $\alpha=1$ and $h(1)=1$ in (10), we get 


$$
\xi(u)-\xi(v) \leq \eta(\xi(u), \xi(v)),
$$

for any $u, v \in I$. (13) obviously implies (12).

We observe that if $\xi: I \longrightarrow \mathbb{R}$ is a convex stochastic process and $\eta: \xi(I) \times \xi(I) \longrightarrow \mathbb{R}$ is an arbitrary bifunction that satisfies

$$
\eta(a, b) \geq a-b
$$

for any $a, b \in I$ and $h(\alpha) \geq \alpha$, then for any $u, v \in I$ and $\alpha \in[0,1]$, we have

$$
\begin{aligned}
\xi(\alpha u+(1-\alpha) v) & \leq \xi(v)+\alpha(\xi(u)-\xi(v)) \\
& \leq \xi(v)+h(\alpha) \eta(\xi(u), \xi(v)),
\end{aligned}
$$

showing that $\xi$ is a generalized $h$-convex stochastic process.

Example 1. Every generalized $h$-convex function gives an example of a generalized $h$-convex stochastic process.

Example 2. Let $\xi: I \times \Omega \longrightarrow \mathbb{R}$ be a convex stochastic process. For every $k \leq 1$, consider the function

$$
\begin{aligned}
h_{k}:(0,1) & \longrightarrow \mathbb{R}, \\
x & \longmapsto x^{k} .
\end{aligned}
$$

Note that $h_{k}(\alpha) \geq \alpha$ for all $\alpha \in(0,1)$. Also, take $\eta(x, y)=x-y$. Moreover, for every $u, v \in I$ and $\alpha \in(0,1)$, the following inequality is satisfied:

$$
\begin{aligned}
\xi(\alpha u+(1-\alpha) v) & \leq \alpha \xi(u)+(1-\alpha) \xi(v) \\
& \leq \xi(v)+h_{k}(\alpha) \eta(\xi(u), \xi(v))(\text { a.e }) .
\end{aligned}
$$

Then, $\xi$ is a generalized $h_{k}$-convex stochastic process.

The paper is organized as follows: in Section 4, we will derive some basic results for the generalized $h$-convex stochastic process. In Section 5, Jensen-type inequality will be proved, whereas in Section 6, Hermite-Hadamard and Fejér-type inequalities will be established. Finally, in Section 7, we will derive Ostrowski-type inequality for generalized $h$-convex stochastic processes.

\section{Basic Results}

Proposition 1. Consider two generalized h-convex stochastic processes $\xi_{1}, \xi_{2}: I \times \Omega \longrightarrow \mathbb{R}$ such that

(1) If $\eta$ is additive, then $\xi_{1}+\xi_{2}: I \longrightarrow \mathbb{R}$ is $\eta_{h}$-convex stochastic process

(2) If $\eta$ is nonnegatively homogeneous, then for any $\gamma \geq 0$, $\gamma \xi_{1}: I \times \Omega \longrightarrow \mathbb{R}$ is a generalized $h$-convex stochastic process

(3) If $\xi_{2}$ is linear, then $\xi_{1} \circ \xi_{2}$ is a generalized $h$-convex stochastic process

Proof. The proof of the proposition is straightforward.

Proposition 2. If $\xi:[a, b] \longrightarrow \mathbb{R}$ is a generalized $h$-convex stochastic process and $h(\alpha) \leq k$, then we have almost everywhere

$$
\max _{u \in[a, b]} \xi(u) \leq \max \{\xi(b), \xi(b)+k \eta(\xi(a), \xi(b))\}
$$

Proof. For any $u \in[a, b]$, we have $u=\alpha a+(1-\alpha) b$, for some $\alpha \in[0,1]$, and

$$
\xi(u)=\xi(\alpha a+(1-\alpha) b) .
$$

since $\xi$ is a generalized $h$-convex stochastic process, so

$$
\begin{aligned}
\xi(u) & \leq \xi(b)+h(\alpha) \eta(\xi(a), \xi(b)) \\
& \leq \max \{\xi(b), \xi(b)+k \eta(\xi(a), \xi(b))\} .
\end{aligned}
$$

Since $u$ is arbitrary,

$$
\max _{u \in[a, b]} \xi(u) \leq \max \{\xi(b), \xi(b)+k \eta(\xi(a), \xi(b))\} \text {. }
$$

\section{Jensen-Type Inequality}

We will use the next lemma to derive the Jensen-type inequality for the generalized $h$-convex stochastic process.

Lemma 1. Assume a generalized $h$-convex stochastic process $\xi: I \times \Omega \longrightarrow \mathbb{R}$. For $u_{1}, u_{2} \in I$ and $\alpha_{1}+\alpha_{2}=1$, we have

$$
\xi\left(\alpha_{1} u_{1}+\alpha_{2} u_{2}\right) \leq \xi\left(u_{2}\right)+h\left(\alpha_{1}\right) \eta\left(\xi\left(u_{2}\right), \xi\left(u_{2}\right)\right)(\text { a.e }) \text {. }
$$

Also, when $n>2$ for $u_{1}, u_{2}, \ldots, u_{n} \in I, \quad \sum_{i=1}^{n} \alpha_{i}=1$, and $T_{i}=\sum_{j=1} \alpha_{j}$, the following inequality holds almost everywhere:

$$
\begin{aligned}
\xi\left(\sum_{i=1}^{n} \alpha_{i} u_{i}\right) & =\xi\left(\left(T_{n-1} \sum_{i=1}^{n-1} \frac{\alpha_{i}}{T_{n-1}} u_{i}\right)+\alpha_{n} u_{n}\right) \\
& \leq \xi\left(u_{n}\right)+h\left(T_{n-1}\right) \eta\left(\xi\left(\sum_{i=1}^{n-1} \frac{\alpha_{i}}{T_{n-1}} u_{i}\right), \xi\left(u_{n}\right)\right) .
\end{aligned}
$$

Theorem 1 (Jensen-type inequality). Assume a generalized $h$-convex stochastic process $\xi: I \times \Omega \longrightarrow \mathbb{R}$, and let $\eta: A \times$ $B \longrightarrow \mathbb{R}$ be nondecreasing, nonnegatively sublinear in the first variable. If $T_{i}=\sum_{j=1}^{i} \alpha_{j}$ for $i=1,2, \ldots, n$ such that $T_{n}=1$, then we have almost everywhere

$$
\xi\left(\sum_{i=1}^{n} \alpha_{i} u_{i}\right) \leq \xi\left(u_{n}\right)+\sum_{i=1}^{n-1} h(T)_{i} \eta_{\xi}\left(u_{i}, u_{i+1}, \ldots, u_{n}\right),
$$

where

$$
\begin{aligned}
\eta_{\xi}\left(u_{i}, u_{i+1}, \ldots, u_{n}\right) & =\eta\left(\eta_{\xi}\left(u_{i}, u_{i+1}, \ldots, u_{n-1}\right),\right. \\
\left.\xi\left(u_{n}\right)\right) \text { and } \eta_{\xi}(u) & =\xi(u),
\end{aligned}
$$

for all $u \in I$.

Proof. Since $\eta$ is nondecreasing, nonnegatively sublinear in the first variable, from Lemma 1, we have 


$$
\begin{aligned}
\xi\left(\sum_{i=1}^{n} \alpha_{i} u_{i}\right) \leq & \xi\left(u_{n}\right)+h\left(T_{n-1}\right) \eta\left(\xi\left(\sum_{i=1}^{n-1} \frac{\alpha_{i}}{T_{n-1}} u_{i}\right), \xi\left(u_{n}\right)\right)=\xi\left(u_{n}\right) \\
& +h\left(T_{n-1}\right) \eta\left(\xi\left(\frac{T_{n-2}}{T_{n-1}} \sum_{i=1}^{n-2} \frac{\alpha_{i}}{T_{n-2}} u_{i}+\frac{\alpha_{n-1}}{T_{n-1}} u_{n-1}\right), \xi\left(u_{n}\right)\right) \\
\leq & \xi\left(u_{n}\right)+h\left(T_{n-1}\right) \eta\left(\xi\left(u_{n-1}\right)+h\left(\frac{T_{n-2}}{T_{n-1}}\right) \times \eta\left(\xi\left(\sum_{i=1}^{n-2} \frac{\alpha_{i}}{T_{n-2}} u_{i}\right), \xi\left(u_{n-1}\right)\right), \xi\left(u_{n}\right)\right) \\
\leq & \xi\left(u_{n}\right)+h\left(T_{n-1}\right) \eta\left(\xi\left(u_{n-1}\right), \xi\left(u_{n}\right)\right) \\
& +h\left(T_{n-2}\right) \eta\left(\eta\left(\xi\left(\sum_{i=1}^{n-2} \frac{\alpha_{i}}{T_{n-2}} u_{i}\right), \xi\left(u_{n-1}\right)\right), \xi\left(u_{n}\right)\right) \leq \ldots \\
\leq & \xi\left(u_{n}\right)+h\left(T_{n-1}\right) \eta\left(\xi\left(u_{n-1}\right), \xi\left(u_{n}\right)\right) \\
& +h\left(T_{n-2}\right) \eta\left(\eta\left(\xi\left(u_{n-2}\right), \xi\left(u_{n-1}\right)\right), \xi\left(u_{n}\right)\right)+\ldots \\
& +h\left(T_{1}\right) \eta\left(\eta\left(\ldots \eta\left(\eta\left(\xi\left(u_{1}\right), \xi\left(u_{2}\right)\right), \xi\left(u_{3}\right)\right) \ldots\right)\right),\left(\xi\left(u_{n-1}\right)\right),\left(\xi\left(u_{n}\right)\right) \\
= & \xi\left(u_{n}\right)+h\left(T_{n-1}\right) \eta_{\xi}\left(u_{n-1}, u_{n}\right)+h\left(T_{n-2}\right) \eta_{\xi}\left(u_{n-2}, u_{n-1}, u_{n}\right)+\ldots+h\left(T_{1}\right) \eta_{\xi}\left(u_{1}, u_{2}, \ldots, u_{n-1}, u_{n}\right) .
\end{aligned}
$$

Finally, small calculations yield (26).

$$
\xi\left(\frac{a_{1}+a_{2}}{2}\right)=\xi\left(\frac{x+y}{2}\right)
$$

\section{Hermite-Hadamard and Fejér- Type Inequalities}

Theorem 2. Assume a mean-square integrable generalized $h$-convex stochastic process $\xi:\left[a_{1}, a_{2}\right] \times \Omega \longrightarrow \mathbb{R}$. Then, for any $a_{1}, a_{2} \in I\left(a_{1}<a_{2}\right)$, the following inequality holds almost everywhere:

$$
\begin{aligned}
& \xi\left(\frac{a_{1}+a_{2}}{2}\right)-\frac{h(1 / 2)}{a_{2}-a_{1}} \int_{a_{1}}^{a_{2}} \eta\left(\xi \left(a_{1}\right.\right. \\
& \left.\left.\quad+a_{2}-u\right), \xi(u)\right) \mathrm{d} u \\
& \leq \frac{1}{a_{2}-a_{1}} \int_{a_{1}}^{a_{2}} \xi(u) \mathrm{d} u \leq \frac{\xi\left(a_{1}\right)+\xi\left(a_{2}\right)}{2} \\
& \quad+\frac{1}{2}\left[\eta\left(\xi\left(a_{1}\right), \xi\left(a_{2}\right)\right)\right. \\
& \left.\quad+\eta\left(\xi\left(a_{2}\right), \xi\left(a_{1}\right)\right)\right] \int_{0}^{1} h(\alpha) \mathrm{d} \alpha .
\end{aligned}
$$

Proof. Let $x=\alpha a_{1}+(1-\alpha) a_{2}$ and $y=(1-\alpha) a_{1}+\alpha a_{2}$; then,

$$
\begin{aligned}
= & \xi\left(\frac{1}{2}\left(\alpha a_{1}+(1-\alpha) a_{2}\right)+\frac{1}{2}\left((1-\alpha) a_{1}+\alpha a_{2}\right)\right) \\
\leq & \xi\left((1-\alpha) a_{1}+\alpha a_{2}\right)+h\left(\frac{1}{2}\right) \\
& \times \eta\left(\xi\left(\alpha a_{1}+(1-\alpha) a_{2}\right), \xi\left((1-\alpha) a_{1}+\alpha a_{2}\right)\right) .
\end{aligned}
$$
$[0,1]$,

Integrating the above inequality with respect to' $\alpha$ ' over

$$
\begin{aligned}
& \xi\left(\frac{a_{1}+a_{2}}{2}\right) \leq \frac{1}{a_{2}-a_{1}} \int_{a_{1}}^{a_{2}} \xi(u) \mathrm{d} u+\frac{h(1 / 2)}{a_{2}-a_{1}} \int_{a_{1}}^{a_{2}} \eta \\
& \cdot\left(\xi\left(a_{1}+a_{2}-u\right), \xi(u)\right) \mathrm{d} u \\
& \xi\left(\frac{a_{1}+a_{2}}{2}\right)-\frac{h(1 / 2)}{a_{2}-a_{1}} \int_{a_{1}}^{a_{2}} \eta\left(\xi\left(a_{1}+a_{2}-u\right),\right. \\
& \xi(u)) \mathrm{d} u \leq \frac{1}{a_{2}-a_{1}} \int_{a_{1}}^{a_{2}} \xi(u) \mathrm{d} u .
\end{aligned}
$$

Now, 


$$
\begin{aligned}
\int_{a_{1}}^{a_{2}} \xi(u) \mathrm{d} u= & \left(a_{2}-a_{1}\right) \int_{0}^{1} \xi\left(\alpha a_{1}+(1-\alpha) a_{2}\right) \mathrm{d} \alpha \\
\leq & \left(a_{2}-a_{1}\right)\left[\xi\left(a_{2}\right)+\int_{0}^{1} h(\alpha) \eta\left(\xi\left(a_{1}\right), \xi\left(a_{2}\right)\right) d \alpha\right] \\
& \frac{1}{a_{2}-a_{1}} \int_{a_{1}}^{a_{2}} \xi(u) \mathrm{d} u \leq \xi\left(a_{2}\right) \\
& +\int_{0}^{1} h(\alpha) \eta\left(\xi\left(a_{1}\right), \xi\left(a_{2}\right)\right) \mathrm{d} \alpha
\end{aligned}
$$

Similarly,

$$
\frac{1}{a_{2}-a_{1}} \int_{a_{1}}^{a_{2}} \xi(u) \mathrm{d} u \leq \xi\left(a_{1}\right)+\int_{0}^{1} h(\alpha) \eta\left(\xi\left(a_{2}\right), \xi\left(a_{1}\right)\right) \mathrm{d} \alpha .
$$

$$
\begin{array}{r}
\frac{1}{a_{2}-a_{1}} \int_{a_{1}}^{a_{2}} \xi(u) \mathrm{d} u \leq \frac{\xi\left(a_{1}\right)+\xi\left(a_{2}\right)}{2}+\frac{1}{2}\left[\eta\left(\xi\left(a_{1}\right), \xi\left(a_{2}\right)\right)+\eta\left(\xi\left(a_{2}\right), \xi\left(a_{1}\right)\right)\right] \int_{0}^{1} h(\alpha) \mathrm{d} \alpha . \\
\xi(x)=\xi(a+b-x),
\end{array}
$$

Combining (29) and (32), we get (27).

for any $a \leq x \leq b$.

Remark 1. If we take $\eta(x, y)=x-y$ and $h(\alpha)=\alpha$, then Theorem 1 reduces to the Hermite-Hadamard inequality for stochastic convexity in [22].

Theorem 3. Assume a generalized h-convex stochastic process $\xi_{1}:\left[a_{1}, a_{2}\right] \times \Omega \longrightarrow \mathbb{R}$ with $\eta$ bounded above on $\xi_{1}\left(\left[a_{1}, a_{2}\right]\right) \times \xi_{1}\left(\left[a_{1}, a_{2}\right]\right)$ and a nonnegative function $h:(0,1) \longrightarrow \mathbb{R}$. Also, suppose that $\xi_{2}:\left[a_{1}, a_{2}\right] \longrightarrow \mathbb{R}^{+}$is integrable and symmetric about $a_{1}+a_{2} / 2$. Then,

Definition 6 (see [36]). A stochatic process $\xi:[a, b] \longrightarrow \mathbb{R}$ i said to be symmetric with respect to $a+b / 2$ on $[a, b]$ if

$$
+\frac{\eta\left(\xi_{1}\left(a_{1}\right), \xi_{1}\left(a_{2}\right)\right)+\eta\left(\xi_{1}\left(a_{2}\right), \xi_{1}\left(a_{1}\right)\right)}{2 h\left(a_{2}-a_{1}\right)} \times \int_{a_{1}}^{a_{2}} h\left(a_{2}-u\right) \xi_{2}(u) \mathrm{d} u .
$$

Proof. By using the definition of the generalized $h$-stochastic convexity of $\xi_{1}$, change of variable, and the assumption that $\xi_{2}$ is symmetric about $a_{1}+a_{2} / 2$, we have

$$
\begin{aligned}
& \int_{a_{1}}^{a_{2}} \xi_{1}(u) \xi_{2}(u,) \mathrm{d} u \leq\left(a_{2}-a_{1}\right) \int_{0}^{1}\left[\xi_{1}\left(a_{2}\right)+h(\alpha) \eta\left(\xi_{1}\left(a_{1}\right), \xi_{1}\left(a_{2}\right)\right)\right] \\
& \times \xi_{2}\left(\alpha a_{1}+(1-\alpha) a_{2}\right) \mathrm{d} \alpha \\
= & \left(a_{2}-a_{1}\right)\left[\int_{0}^{1} \xi_{1}\left(a_{2}\right) \xi_{2}\left(\alpha a_{1}+(1-\alpha) a_{2}\right) \mathrm{d} \alpha+\eta\left(\xi\left(a_{1}\right), \xi\left(a_{2}\right)\right) \int_{0}^{1} h(\alpha) \xi_{2}\left(\alpha a_{1}+(1-\alpha) a_{2}\right) \mathrm{d} \alpha\right], \\
\int_{a_{1}}^{a_{2}} \xi_{1}(u,) \xi_{2}(u,) \mathrm{d} u \leq & \left(a_{2}-a_{1}\right) \int_{0}^{1}\left[\xi_{1}\left(a_{1}\right)+h(\alpha) \eta\left(\xi_{1}\left(a_{2}\right), \xi_{1}\left(a_{1}\right)\right)\right] \\
& \times \xi_{2}\left((1-\alpha) a_{1}+\alpha a_{2}\right) \mathrm{d} \alpha=\left(a_{2}-a_{1}\right)\left[\int_{0}^{1} \xi_{1}\left(a_{1}\right) \xi_{2}\left(\alpha a_{1}+(1-\alpha) a_{2}\right) \mathrm{d} \alpha\right. \\
& \left.+\eta\left(\xi_{1}\left(a_{2}\right), \xi_{1}\left(a_{1}\right)\right) \int_{0}^{1} h(\alpha) \xi_{2}\left(\alpha a_{1}+(1-\alpha) a_{2}\right) \mathrm{d} \alpha\right]
\end{aligned}
$$

Adding (35) and (36), we get 


$$
\begin{aligned}
& 2 \int_{a_{1}}^{a_{2}} \xi_{1}(u) \xi_{2}(u) \mathrm{d} u \leq\left(a_{2}-a_{1}\right)\left(\xi_{1}\left(a_{1}\right)+\xi_{1}\left(a_{2}\right)\right) \\
& \times \int_{0}^{1} \xi_{2}\left(\alpha a_{1}+(1-\alpha) a_{2}\right) \mathrm{d} \alpha+\left(a_{2}-a_{1}\right)\left(\eta\left(\xi_{1}\left(a_{1}\right), \xi_{1}\left(a_{2}\right)\right)\right) \\
& +\eta\left(\left(\xi_{1}\left(a_{2}\right), \xi_{1}\left(a_{1}\right)\right)\right) \int_{0}^{1} h(\alpha) \xi_{2}\left(\alpha a_{1}+(1-\alpha) a_{2}\right) \mathrm{d} \alpha
\end{aligned}
$$

and by changing the variable $u=\alpha a_{1}+(1-\alpha) a_{2}$, we obtain

$$
\begin{aligned}
& \int_{a_{1}}^{a_{2}} \xi_{1}(u) \xi_{2}(u) \mathrm{d} u \leq \frac{\xi_{1}\left(a_{1}\right)+\xi_{1}\left(a_{2}\right)}{2} \\
& \cdot \int_{a_{1}}^{a_{2}} \xi_{2}(u) \mathrm{d} u \\
& +\frac{\eta\left(\xi_{1}\left(a_{1}\right), \xi_{1}\left(a_{2}\right)\right)+\eta\left(\xi_{1}\left(a_{2}\right), \xi_{1}\left(a_{1}\right)\right)}{2 h\left(a_{2}-a_{1}\right)} \\
& \quad \times \int_{a_{1}}^{a_{2}} h\left(a_{2}-u\right) \xi_{2}(u) \mathrm{d} u .
\end{aligned}
$$

Theorem 4. Assume a generalized $h$-convex stochastic process $\xi_{1}:\left[a_{1}, a_{2}\right] \times \Omega \longrightarrow \mathbb{R}$ with $\eta$ bounded above on $\xi_{1}\left(\left[a_{1}, a_{2}\right]\right) \times \xi_{1}\left(\left[a_{1}, a_{2}\right]\right)$ and a nonnegative function $h:(0,1) \longrightarrow \mathbb{R}$. Also, suppose that $\xi_{2}:\left[a_{1}, a_{2}\right] \longrightarrow \mathbb{R}^{+}$is integrable and symmetric about $a_{1}+a_{2} / 2$. Then,

$$
\begin{aligned}
& \xi_{1}\left(\frac{a_{1}+a_{2}}{2}\right) \int_{a_{1}}^{a_{2}} \xi_{2}(u)-h\left(\frac{1}{2}\right) \\
& \cdot \int_{a_{1}}^{a_{2}} \eta\left(\xi_{1}\left(a_{1}+a_{2}-u\right), \xi(u)\right) \xi_{2}(u) \mathrm{d} u \\
& \quad \leq \int_{a_{1}}^{a_{2}} \xi(u) \xi_{2}(u) \mathrm{d} u .
\end{aligned}
$$

Proof. Using the definition of the generalized $h$-convex stochastic process, change of variable, and the assumption that $\xi_{2}$ is symmetric about $a_{1}+a_{2} / 2$, we get

$$
\begin{aligned}
\xi_{1}\left(\frac{a_{1}+a_{2}}{2}\right) & =\xi_{1}\left(\frac{\alpha a_{1}-\alpha a_{1}+a_{1}+a_{2}+\alpha a_{2}-\alpha a_{2}}{2}\right) \\
& =\xi_{1}\left(\frac{\alpha a_{1}+(1-\alpha) a_{2}+\alpha a_{2}+(1-\alpha) a_{1}}{2}\right) \\
& \leq \xi_{1}\left(\alpha a_{2}+(1-\alpha) a_{1}\right)+h\left(\frac{1}{2}\right) \\
& \times \eta\left(\xi_{1}\left(\alpha a_{2}+(1-\alpha) a_{1}\right), \xi_{1}\left(\alpha a_{2}+(1-\alpha) a_{1}\right)\right) .
\end{aligned}
$$

By changing the variable $u=\alpha a_{2}+(1-\alpha) a_{1}$, we obtain

$$
\begin{aligned}
& \xi_{1}\left(\frac{a_{1}+a_{2}}{2}\right) \int_{a_{1}}^{a_{2}} \xi_{2}(u) \mathrm{d} u=\xi_{1}\left(\frac{a_{1}+a_{2}}{2}\right) \\
& \quad \cdot \int_{0}^{1} \xi_{2}\left(\alpha a_{2}+(1-\alpha) a_{1}\right)\left(a_{2}-a_{1}\right) \mathrm{d} \alpha \\
& \leq \int_{0}^{1} \xi_{1}\left(\alpha a_{2}+(1-\alpha) a_{1}\right) \xi_{2}\left(\alpha a_{2}\right. \\
& \left.\quad+(1-\alpha) a_{1}\right)\left(a_{2}-a_{1}\right) \mathrm{d} \alpha \\
& \quad+h\left(\frac{1}{2}\right) \int_{0}^{1} \eta\left(\xi_{1}\left(\alpha a_{1}+(1-\alpha) a_{2}\right), \xi_{1}\left(\alpha a_{2}+(1-\alpha) a_{1}\right)\right) \\
& \quad \times \xi_{2}\left(\alpha a_{2}+(1-\alpha) a_{1}\right)\left(a_{2}-a_{1}\right) \mathrm{d} \alpha \\
& =\int_{a_{1}}^{a_{2}} \xi_{1}(u) \xi_{2}(u) \mathrm{d} u+h\left(\frac{1}{2}\right) \\
& \quad \times \int_{a_{1}}^{a_{2}} \eta\left(\xi_{1}\left(a_{1}+a_{2}-u\right), \xi_{1}(u)\right) \xi_{2}(u) \mathrm{d} u .
\end{aligned}
$$

Corollary 1. By setting $\eta(x, y)=x-y$ and $h(\alpha)=\alpha$ in (34 and 39) and then combining, we obtain the classical Hermite-Hadamard-Fejér-type inequality as

$$
\begin{aligned}
\xi_{1}\left(\frac{a_{1}+a_{2}}{2}\right) \int_{a_{1}}^{a_{2}} \xi_{2}(u) \mathrm{d} u \leq & \int_{a_{1}}^{a_{2}} \xi_{1}(u) \xi_{2}(u) \mathrm{d} u \\
\leq & \frac{\xi_{1}\left(a_{1}\right)+\xi_{1}\left(a_{2}\right)}{2} \\
& \cdot \int_{a_{1}}^{a_{2}} \xi_{2}(u) \mathrm{d} u .
\end{aligned}
$$

\section{Ostrowski-Type Inequality}

In order to prove Ostrowski-type inequality for the generalized $h$-convex stochastic process, the following lemma is required.

Lemma 2 (see [37]). Let $\xi: I \times \Omega \longrightarrow \mathbb{R}$ be a stochastic process which is mean-square differentiable on $I^{\circ}$ and its derivative $\xi^{\prime}$ be mean-square integrable on $\left[a_{1}, a_{2}\right]$, where $a_{1}$, $a_{2} \in I$ with $a_{1}<a_{2}$; then, the following equality holds:

$$
\begin{aligned}
\xi(u) & -\frac{1}{a_{2}-a_{1}} \int_{a_{1}}^{a_{2}} \xi(v) \mathrm{d} v=\frac{\left(u-a_{1}\right)^{2}}{a_{2}-a_{1}} \\
\cdot & \int_{0}^{1} \alpha \xi^{\prime}\left(\alpha u+(1-\alpha) a_{1}\right) \mathrm{d} \alpha \\
& -\frac{\left(a_{2}-u\right)^{2}}{a_{2}-a_{1}} \int_{0}^{1} \alpha \xi^{\prime}\left(\alpha u+(1-\alpha) b_{1}\right) \mathrm{d} \alpha(\text { a.e. }),
\end{aligned}
$$

for each $u \in[a, b]$. 
Theorem 5. Assume a mean-square stochastic process $\xi: I \times$ $\Omega \longrightarrow \mathbb{R}$ such that $\xi$ । (the derivative of $\xi$ ) is mean-square integrable on $\left[a_{1}, a_{2}\right]$, where $a_{1}, a_{2} \in I$ with $a_{1}<a_{2}$, and consider a nonnegative function $h:(0,1) \longrightarrow \mathbb{R}$ which is supermultiplicative such that, for every $\alpha, h(\alpha)>\alpha$. If $|\xi \prime|$ is a generalized h-convex stochastic process on $I$ and $\left|\xi_{\prime}(u)\right| \leq M$ for every $u$, then

$$
\begin{aligned}
\left|\xi(u)-\frac{1}{a_{2}-a_{1}} \int_{a_{1}}^{a_{2}} \xi(v) \mathrm{d} v\right| \leq & M\left[\frac{\left(u-a_{1}\right)^{2}+\left(a_{2}-u\right)^{2}}{a_{2}-a_{1}}\right] \int_{0}^{1} h(y) \mathrm{d} y \\
& +\left(\frac{\left(u-a_{1}\right)^{2}}{a_{2}-a_{1}} \eta\left(\left|\xi^{\prime}(u)\right|,\left|\xi^{\prime}\left(a_{1}\right)\right|\right)+\frac{\left(a_{2}-u\right)^{2}}{a_{2}-a_{1}} \eta\left(\left|\xi^{\prime}(u)\right|,\left|\xi^{\prime}\left(a_{2}\right)\right|\right)\right) \int_{0}^{1} h^{2}(y) \mathrm{d} y .
\end{aligned}
$$

Proof. By using Lemma 2 and the definition of generalized $h$-stochastic convexity of $\left|\xi^{\prime}\right|$, we get

$$
\begin{aligned}
& \left|\xi(u)-\frac{1}{a_{2}-a_{1}} \int_{a_{1}}^{a_{2}} \xi(v) \mathrm{d} v\right| \leq \frac{\left(u-a_{1}\right)^{2}}{a_{2}-a_{1}} \int_{0}^{1} y\left|\xi \prime\left(y u+(1-y) a_{1}\right)\right| \mathrm{d} y \\
& +\frac{\left(a_{2}-u\right)^{2}}{a_{2}-a_{1}} \int_{0}^{1} y\left|\xi^{\prime}\left(y u+(1-y) a_{2}\right)\right| \mathrm{d} y \\
& \leq \frac{\left(u-a_{1}\right)^{2}}{a_{2}-a_{1}} \int_{0}^{1} y\left[\left|\xi_{\prime}\left(a_{1}\right)\right|+h(y) \eta\left(\left|\xi_{\prime}(u)\right|,\left|\xi_{\prime}\left(a_{1}\right)\right|\right)\right] \mathrm{d} y \\
& +\frac{\left(a_{2}-u\right)^{2}}{a_{2}-a_{1}} \int_{0}^{1} y\left[\left|\xi_{\prime}\left(a_{2}\right)\right|+h(y) \eta\left(\left|\xi_{\prime}(u)\right|,\left|\xi_{\prime}\left(a_{2}\right)\right|\right)\right] \mathrm{d} y \leq M \frac{\left(u-a_{1}\right)^{2}}{a_{2}-a_{1}} \int_{0}^{1} h(y) \mathrm{d} y \\
& +\frac{\left(u-a_{1}\right)^{2}}{a_{2}-a_{1}} \int_{0}^{1} h^{2}(y) \eta\left(\left|\xi_{\prime}(u)\right|,\left|\xi_{\prime}\left(a_{1}\right)\right|\right) \mathrm{d} y+M \frac{\left(a_{2}-u\right)^{2}}{a_{2}-a_{1}} \int_{0}^{1} h(y) \mathrm{d} y \\
& +\frac{\left(a_{2}-u\right)^{2}}{a_{2}-a_{1}} \int_{0}^{1} h^{2}(y) \eta\left(\left|\xi_{\prime}(u)\right|,\left|\xi_{\prime}\left(a_{2}\right)\right|\right) \mathrm{d} y \leq M\left[\frac{\left(u-a_{1}\right)^{2}+\left(a_{2}-u\right)^{2}}{a_{2}-a_{1}}\right] \int_{0}^{1} h(y) \mathrm{d} y \\
& +\frac{\left(u-a_{1}\right)^{2}}{a_{2}-a_{1}} \eta\left(\left|\xi_{\prime}(u)\right|,\left|\xi_{\prime}\left(a_{1}\right)\right|\right) \times \int_{0}^{1} h^{2}(y) \mathrm{d} y+\frac{\left(a_{2}-u\right)^{2}}{a_{2}-a_{1}} \eta\left(\left|\xi_{\prime}(u)\right|,\left|\xi_{\prime}\left(a_{2}\right)\right|\right) \int_{0}^{1} h^{2}(y) \mathrm{d} y \\
& =M\left[\frac{\left(u-a_{1}\right)^{2}+\left(a_{2}-u\right)^{2}}{a_{2}-a_{1}}\right] \int_{0}^{1} h(y) \mathrm{d} y+\left(\frac{\left(u-a_{1}\right)^{2}}{a_{2}-a_{1}} \eta\left(\left|\xi_{\prime}(u)\right|,\left|\xi_{\prime}\left(a_{1}\right)\right|\right)\right. \\
& \left.+\frac{\left(a_{2}-u\right)^{2}}{a_{2}-a_{1}} \eta\left(\left|\xi_{\prime}(u)\right|,\left|\xi_{\prime}\left(a_{2}\right)\right|\right)\right) \int_{0}^{1} h^{2}(y) \mathrm{d} y .
\end{aligned}
$$

\section{Conclusion}

Stochastic processes have many applications in statistics, which obviously lead to lots of other domains, for example, Kolmogorov-Smirnoff test on the equality of distributions [38-40] (the test statistic is derived from a Brownian bridge, which is a Brownian motion conditioned to have certain values at the endpoints of an interval of time). The other applications include sequential analysis [41, 42] (this is the rigorous way you can stop an A/B test dynamically. Stopping rules are obtained by approximating discrete problems with their continuous time analogs, in which the sufficient statistic process follows a stochastic differential equation) and quickest detection $[43,44]$ (my stock story is that you are using a gold mine which gives you a random amount of gold per day, but at some point, it will get depleted, and you want to know when this happens as quickly as possible. Decision rules are again based on properties of hitting times of 
random processes). In this paper, we have introduced generalized $h$-conevex stochastic processes and proved Jensen, Hermite-Hadamard, and Fejér-type inequalities. Our results are applicable because applying the convex function to the expected value of a random variable is always bounded above by the expected value of the convex function of the random variable.

\section{Data Availability}

All data required for this research are included within this paper.

\section{Conflicts of Interest}

The authors do not have any conflicts of interest.

\section{Authors' Contributions}

All authors contributed equally to this paper.

\section{Acknowledgments}

This research was supported by the National Natural Science Foundation of China (Grant nos. 11971142, 11871202, 61673169, 11701176, 11626101, and 11601485).

\section{References}

[1] S. Ghahramani, Fundamentals of Probability with Stochastic Process, Pearson Education India, New York, NY, USA, 2005.

[2] A. Anguraj and R. Kumar, "Existence and uniqueness of mild solution for stochastic partial differential equation with Poisson jumps and delays," Open Journal of Mathematical Sciences, vol. 3, no. 1, pp. 343-348, 2019.

[3] A. Kumar, "Optimal control analysis of combined anti-angiogenic and tumor immunotherapy," Open Journal of Mathematical Sciences, vol. 3, no. 1, pp. 349-357, 2019.

[4] K. Itô, "On stochastic processes (I)," in Japanese Journal of Mathematics: Transactions and Abstracts, vol. 18, pp. 261-301, The Mathematical Society of Japan, Japan, 1941.

[5] H. P. Hong, "Application of the stochastic process to pitting corrosion," Corrosion, vol. 55, no. 1, pp. 10-16, 1999.

[6] J. L. Doob, "Stochastic processes depending on a continuous parameter," Transactions of the American Mathematical Society, vol. 42, no. 1, p. 107, 1937.

[7] M. Shaked and J. G. Shanthikumar, "Parametric stochastic convexity and concavity of stochastic processes," Annals of the Institute of Statistical Mathematics, vol. 42, no. 3, pp. 509-531, 1990.

[8] P. Chen, A. Quarteroni, and G. Rozza, "Stochastic optimal Robin boundary control problems of advection-dominated elliptic equations," SIAM Journal on Numerical Analysis, vol. 51, no. 5, pp. 2700-2722, 2013.

[9] A. Bogdanov, S. Chiu, L. U. Gokdere, and J. Vian, "Stochastic optimal control of a servo motor with a lifetime constraint," in Proceedings of the 45th Ieee Conference on Decision and Control, IEEE, Berlin, Germany, pp. 4182-4187, 2006.

[10] A. Belloni, L. Piroddi, and M. Prandini, "A stochastic optimal control solution to the energy management of a microgrid with storage and renewables," in Proceedings of the 2016 American Control Conference (ACC), IEEE, Berlin, Germany, pp. 2340-2345, 2016.
[11] S. Haesaert, P. M. Van den Hof, and A. Abate, "Experiment design for formal verification via stochastic optimal control," in Proceedings of the 2016 European Control Conference (ECC), IEEE, Berlin, Germany, pp. 427-432, 2016.

[12] I. Exarchos, E. A. Theodorou, and P. Tsiotras, "Game-theoretic and risk-sensitive stochastic optimal control via forward and backward stochastic differential equations," in Proceedings of the 2016 IEEE 55th Conference on Decision and Control (CDC), IEEE, Berlin, Germany, pp. 6154-6160, 2016.

[13] A. W. Roberts, "Convex functions," in Handbook of Convex Geometry, pp. 1081-1104, IEEE, North-Holland, MA, USA, 1993.

[14] S. Varošanec, "On h-convexity," Journal of Mathematical Analysis and Applications, vol. 326, no. 1, pp. 303-311, 2007.

[15] M. E. Gordji, M. R. Delavar, and M. D. L. Sen, "On $\varphi$-convex functions," Journal of Mathematical Inequalities, vol. 10, no. 1, pp. 173-183, 2016.

[16] B. Nagy, "On a generalization of the cauchy equation," Aequationes Mathematicae, vol. 10, no. 2-3, pp. 165-171, 1974.

[17] K. Nikodem, "On convex stochastic processes," Aequationes Mathematicae, vol. 20, no. 1, pp. 184-197, 1980.

[18] A. Skowronski, "On some properties of $j$-convex stochastic processes," Aequationes Mathematicae, vol. 44, no. 2-3, pp. 249-258, 1992.

[19] A. Skowronski, "On wright-convex stochastic processes," Aequationes Mathematicae, vol. 9, pp. 29-32, 1995.

[20] Z. Páles, "Nonconvex functions and separation by power means," Mathematical Inequalities \& Applications, vol. 3, no. 2, pp. 169-176, 2000.

[21] R. Rasuli, "Intuitionistic fuzzy subgroups with respect to norms (T,S)," Engineering and Applied Science Letter, vol. 3, no. 2, pp. 40-53, 2020.

[22] D. Kotrys, "Hermite-hadamard inequality for convex stochastic processes," Aequationes Mathematicae, vol. 83, no. 12, pp. 143-151, 2012.

[23] A. Rakhlin, O. Shamir, and K. Sridharan, "Making gradient descent optimal for strongly convex stochastic optimization," 2012.

[24] H. Jin, Z. Q. Xu, and X. Y. Zhou, "A convex stochastic optimization problem arising from portfolio selection," Mathematical Finance, vol. 18, no. 1, pp. 171-183, 2008.

[25] J. Cvitanic and I. Karatzas, "Convex duality in constrained portfolio optimization," The Annals of Applied Probability, vol. 2, no. 4, pp. 767-818, 1992.

[26] H. Kushner and G. Yin, Stochastic Approximation and Recursive Algorithms and Applications, Springer, Berlin, Germany, 2nd edition, 2003.

[27] S. Shalev-Shwartz, O. Shamir, N. Srebro, and K. Sridharan, "Stochastic convex optimization," COLT, Berlin, Germany, 2009.

[28] S. Mehmood and G. Farid, "Fractional integrals inequalities for exponentially $\backslash(\mathrm{m} \backslash)$-convex functions," Open Journal of Mathematical Sciences, vol. 4, no. 1, pp. 78-85, 2020.

[29] W. Farid, K. M. Awan, K. M. Awan, and A. U. Rehman, "An extension of Petrović's inequality for $\backslash(\mathrm{h}-\mathrm{I})$ convex $(\backslash(\mathrm{h}-\mathrm{I})$ concave) functions in plane," Open Journal of Mathematical Sciences, vol. 3, no. 1, pp. 398-403, 2019.

[30] S. I. Farid, M. Nadeem, and G. Farid, "On caputo fractional derivatives via exponential (sm)-convex functions," Engineering and Applied Science Letter, vol. 3, no. 2, pp. 32-39, 2020.

[31] S. Mehmood, G. Farid, K. A. Khan, and M. Yussouf, "New fractional hadamard and fejr-hadamard inequalities 
associated with exponentially (hm)-convex functions," Engineering and Applied Science Letter, vol. 3, no. 2, pp. 9-18, 2020.

[32] Z. Brzezniak and T. Zastawniak, Basic Stochastic Processes: A Course through Exercises, Springer Science \& Business Media, Berlin, Germany, 2000.

[33] K. Sobczyk, Stochastic Differential Equations: with Applications to Physics And Engineering, Springer Science \& Business Media, Berlin, Germany, 2013.

[34] A. V. Skorokhod, "On a generalization of stochastic integral," Teoriya Veroyatnostei I Ee Primeneniya, vol. 20, no. 2, pp. 223-238, 1975.

[35] D. Barráez, L. González, N. Merentes, and A. Moros, "On $h$ convex stochastic processes," Mathematica Aeterna, vol. 5, no. 4, pp. 571-581, 2015.

[36] M. R. Delavar and S. S. Dragomir, "On $\eta$-convexity," Mathematical Inequalities \& Applications, vol. 20, no. 1, pp. 203-216, 2017.

[37] L. Gonzales, J. Materano, and M. V. Lopez, "Ostrowski-type inequalities via hconvex stochastic processes," JP Journal of Mathematical Sciences, vol. 16, no. 2, pp. 15-29, 2016.

[38] S. Engmann and D. Cousineau, "Comparing distributions: the two-sample anderson-darling test as an alternative to the kolmogorov-smirnoff test," Journal of Applied Quantitative Methods, vol. 6, no. 3, pp. 1-17, 2011.

[39] B. B. Bhattacharya, "Two-sample tests based on geometric graphs: asymptotic distribution and detection thresholds," 2015.

[40] R. Dey, "Hypothesis tests with precedence probabilities and precedence-type tests," Wiley Interdisciplinary Reviews: Computational Statistics, vol. 10, no. 1, p. e1417, 2018.

[41] D. Siegmund, Sequential Analysis: Tests and Confidence Intervals, Springer Science \& Business Media, Berlin, Germany, 2013.

[42] P. K. Andersen, O. Borgan, R. D. Gill, and N. Keiding, Statistical Models Based on Counting Processes, Springer Science \& Business Media, Berlin, Germany, 2012.

[43] A. Delorme, T. Sejnowski, and S. Makeig, "Enhanced detection of artifacts in EEG data using higher-order statistics and independent component analysis," Neuroimage, vol. 34, no. 4, pp. 1443-1449, 2007.

[44] P. Dollár, R. Appel, S. Belongie, and P. Perona, "Fast feature pyramids for object detection," IEEE Transactions on Pattern Analysis and Machine Intelligence, vol. 36, no. 8, pp. 15321545, 2014. 